shown by immune agglutinins cannot be ascribed simply to the effect of the viscosity of the serum.

The differentiation of natural and immune agglutinins by their behaviour in agglutination tests suggests that there is a qualitative difference between naturally occurring and immune agglutinins.

In suspected cases of hæmolytic disease of the newborn, the finding of $R h$ agglutinins in the maternal serum strongly supports the clinical diagnosis, but their presence in a serum is often doubtful when the degree of agglutination is very weak. In several instances during the last two years, we have used the agglutination enhancement test and have established beyond doubt the presence of $R h$ agglutinins in such weakly reacting sera. Also, in cases suspected of having received an incompatible blood transfusion, an anti- $A$ or anti- $B$ titre in the recipient's serum which is increased by the use of this technique is a strong indication that the antibody has been stimulated by immunization and that blood of an incompatible $A, B, 0$ group has in fact been given.

A detailed account of the work described in this communication will appear elsewhere.

K. E. Boorman.

B. E. DoDd.

S.W. Blood Transfusion Depot, Sutton.

W. T. J. Morgan.

The Lister Institute of Preventive Medicine, London, S.W.I. July 11.

\section{Transition of Fibrinogen to Fibrin as a Two-Step Reaction}

IT has been assumed by Apitz ${ }^{1}$ that the transition of fibrinogen to fibrin is not a simple reaction, but takes place in two stages. The primary product should be a soluble substance, less stable than fibrinogen, which he called profibrin. By suitable choice of the composition of the electrolytes of the medium, we succeeded in studying the two phases of the reaction separately ; thus we obtained a substance which has a better-founded claim to the name profibrin.

At a $p H$ on the acid side of the is -electric point of fibrinogen, for example, at $p \mathbf{H} 5 \cdot \mathbf{l}$, the addition of thrombin to a solution of fibrinogen does not cause clotting. If now the system is neutralized, say $p H \mathbf{H} 6 \cdot 8$, the fibrinogen eoagulates, which shows that at the acid reaction it has not been irreversibly damaged.

If the neutralization is not carried out immediately, but after a longer reaction at $p \mathrm{H} 5 \cdot 1$, then the clottingtime after neutralization is the shorter the longer fibrinogen and thrombin have been kept together at the acid $p \mathrm{H}$ (see table) :

Composition of the system : $0.5 \mathrm{~cm}^{3}$ flbrinogen according to Laki*, 20 mgm. per cm. . $^{3} 0.9$ per cent sodium chloride; $0.5 \mathrm{~cm} .^{3} M / 5$ potassium dihydrogen phosphate ; $0.1 \mathrm{~cm}^{3}$ thrombin. After different with $0.05 \mathrm{~cm}^{3} M / 5$ disodium hydrogen phosphate. Reaction time

$$
\begin{array}{r}
10 \text { sec. } \\
1 \text { min. } \\
10 \text { " ", } \\
30 \quad " \\
60 \text { " } \\
90 \text { ", } \\
120 \text { ", }
\end{array}
$$

Clotting time:

$6 \mathrm{~min}$.

5

4 min. 30 sec.

3 " 30 "

40

$10 "$,

* Laki, Z. physiol. Chem., 273, 95 (1942). Stud. Inst. Med. Chem. Szeged, 2, 27 (1943).

This shows that the formation of fibrin takes place in two steps; the first step, the primary reaction between fibrinogen and thrombin, also takes place at $p \mathrm{H}$-values on the acid side of the iso-electric point of the former ; the polymerization of the primary product to fibrin, however, is possible only then, when the fibrinogen is negatively charged.

At $p \mathrm{H} \mathbf{5 \cdot 1}$ the presence of thrombin does not cause any physical change of the fibrinogen which could be considered as an indication that polymerization was beginning. The viscosity at a very low velocity-gradient shows only a slight increase ${ }^{2}$.

The second phase of the transition is inhibited by neutral salts in such a way that we conclude that the polymerization of the primary product to fibrin is caused by electrostatic attraction. The first reaction is not inhibited by electrolytes; its nature is still obscure.

K. LAKI.

W. F. H. M. Mommaerts.

Institute of Medical Chemistry, Szeged.

(Prepared for publication: September, 1944.) Now at

The American University, Beirut.

${ }^{1}$ Apitz, Z. exp. Med., 101, 552 (1937).

2 Pittoni, unpublished results.

\section{Effect of Restriction of Food Intake upon the Incisor Teeth of Rachitic Rats}

IN previous publications ${ }^{1}$, it has been shown that the upper incisor teeth of rats are very sensitive indexes of calcium and phosphorus metabolism. Thus in rats on the Steenbock and Black rachitogenic diet No. $2965^{2}$, doses of vitamin D cause a response which, so far as present work goes, seems to be specific to this substance. A line of newly calcified dentin occurs in the proximal labial predentin formed after dosage, but little change takes place in that already formed (Fig. 1). This reaction is accompanied by the usual epiphyseal healing made use of in the line test. Changing the $\mathrm{Ca}: \mathrm{P}$ ratio of the diet given to rachitic rats from $5 \cdot 6$ to $1 \cdot 9$ also causes healing in the teeth and bones, but quite unlike that after vitamin $D$ medication. In the teeth, irregular clumps of calco. spherites are laid down promiscuously in the proximal labial predentin. In the bones, an irregular healing occurs in the epiphysis, usually consisting of up. growths of calcified material in the metaphysis, joining with that in the diaphysis, and often no clear 'line' occurs at all ${ }^{3}$.

It appeared to be of interest to compare these changes with those occurring during restriction of food intake, which is well known to cause healing of rickets in rachitic animals. Three litters of rats were kept on the Steenbock and Black diet until they had well-marked rickets. Some were then dosed with 27 r.U. of vitamin D in oil by mouth and maintained on the same diet. The rest were also kept on the rachitogenic diet, but their food intake was so restricted that they lost on an average $3.6 \mathrm{gm}$. in weight per day. Animals treated in either way were killed and examined on the first, second, fourth, sixth or eighth days after dosage or food restriction. In two of the litters the epiphyses of the restricted rats showed new calcification on the second day, and on the fourth day in the third litter. The changes were quite typical of those seen after vitamin D dosage, with lines forming on the metaphyseal side 